\title{
Incidencia de dispositivos móviles en la educación en el
}

\section{Ecuador}

\section{Incidence of mobile devices in education in Ecuador}

Guillermo Terán Acosta. ${ }^{1}$, Verónica Oña Gamboa. ${ }^{2}$, Juan Carlos Cobos Velasco. ${ }^{3}$ \& Danilo Miniguano Miniguano. ${ }^{4}$

\section{Recibido: 23-06-2019 / Revisado: 28-07-209 /Aceptado: 10-08-2019/ Publicado: 10-09-2019}

\section{Abstract DOI: https://doi.org/10.33262/cienciadigital.v3i3.4.835}

The aim of this paper is to provide systematic data on the incidence of mobile learning or $\mathrm{M}$ in the teaching-learning process.

According to a study GuiaLocal.com, the use of mobile devices grew $61 \%$ in Latin America, while the use of computers to connect to the Internet recorded a fall of $11.3 \%$. Ecuador occupies the 5th place in the statistics of use of mobile devices with a $75 \%$ annual growth.

According to the latest data from the National Institute of Statistics and Census (INEC), $29.1 \%$ of the population use mobile devices for learning and education. According to the Ministry of Education since 2014, regulates the use of cell phones in the classroom level, because the teacher is responsible for authorizing the use of cell in scheduled activities. The use of mobile devices being promoted as a teaching tool, since it is a methodology that complements the learning process, through mobile devices, laptops, tablets, smart mobile phones in this way it seeks to increase the motivation and willingness to learning through the use of mobile devices in education.

Keywords: Mobile, teaching-learning process Information Technology and Communication, education

\section{Resumen}

El objetivo del presente trabajo es ofrecer datos sistemáticos sobre la incidencia de dispositivos móviles o M-learning en la educación en el Ecuador.

\footnotetext{
${ }^{1}$ Facultad de Filosofía, Letras y Ciencias de la Educación, Universidad Central del Ecuador, grteran@uce.edu.ec

${ }^{2}$ Universidad Técnica de Ambato, verona90957@gmail.com

${ }^{3}$ Facultad de Filosofía, Letras y Ciencias de la Educación, Universidad Central del Ecuador, jccobos@uce.edu.ec

${ }^{4}$ Instituto Superior Tecnológico Sucre, dminiguano@tecnologicosucre.edu.ec
} 
Según un estudio de GuiaLocal.com, el uso de dispositivos móviles creció $61 \%$ en países de América Latina, mientras que el uso de computadores para conectarse a Internet registra una caída de $11.3 \%$. Ecuador ocupa el 5to lugar en las estadísticas de uso de dispositivos móviles con un $75 \%$ de crecimiento anual.

Según los últimos datos del Instituto Nacional de Estadística y Censos (INEC), el 29,1\% de la población utiliza los dispositivos móviles para el aprendizaje y la educación. Según el MinEduc desde el año 2014, regula el uso de celulares en el plano pedagógico, ya que el docente es responsable de autorizar el uso del celular dentro de actividades programadas. El uso de dispositivos móviles se promueve como herramienta pedagógica, dado que es una metodología que complementa el proceso de aprendizaje, a través de instrumentos móviles, computadoras portátiles, tablets, teléfonos inteligentes de esta manera se busca incrementar la motivación y la predisposición para el aprendizaje.

Palabras Claves: Dispositivos móviles, educación, Tecnologías de la Información y Comunicación, internet, m-learning

\section{INTRODUCCIÓN}

Como se puede observar nos encontramos cada día inmersos en una sociedad basada en información y conocimiento (Guaña-Moya, 2018). Podemos observar que existen una gran variedad de dispositivos móviles, estos dispositivos móviles inteligentes tienen la capacidad de ejecutar aplicaciones, lo que ha propiciado diversos fenómenos sumamente interesantes, entre estos que los docentes ven la necesidad de actualizarse para aprovechar eficientemente todo el potencial que favorecen en el aprendizaje (Luna-Echeverría, 2018). Esta sociedad denominada, "Sociedad del Conocimiento", se encuentra sometida a constantes cambios y trasformaciones debido al rápido crecimiento tecnológico, promoviendo que se mueva a gran velocidad y de esta manera exige a los individuos un proceso de aprendizaje continuo ya que se han convertido en medios de acceso al conocimiento (Cantillo Valero, Roura Redondo, \& Sanchéz Palacín, 2012), (Pérez-Fabara, 2017).

Los dispositivos móviles representan una nueva oportunidad de acceso a la información por parte de los usuarios. Sin embargo, la diversidad de características de hardware y software que presentan estos dispositivos complica la definición misma del término dispositivo móvil. Por ello, en este artículo se tratará de clarificar este concepto enumerando las características genéricas que todo dispositivo móvil debe tener, también conoceremos los tipos de 
dispositivos móviles existentes con un enfoque histórico para que entender cómo han ido evolucionando estos dispositivos.

A partir del uso de estos recursos se genera un nuevo aprendizaje llamado El M-learning (aprendizaje en movimiento), es un tipo de aprendizaje que tiene como principal característica el uso de dispositivos móviles, para favorecer y apoyar el ámbito educativo en el proceso enseñanza-aprendizaje.

Como resultado de esta problemática el Ecuador se demuestran que el uso de dispositivos móviles se consolida fuertemente, representando un $25.9 \%$ del tráfico total de Internet y generando la necesidad de adaptarse a las nuevas tecnologías.

Por consiguiente, el objetivo de esta investigación es caracterizar los dispositivos móviles en la educación mediante la recopilación de información de esta manera obtener una descripción sobre la incidencia de los distintos dispositivos móviles en la educación.

\section{MÉTODOS}

Se realizó una búsqueda bibliográfica en la web la cual permitió recopilar gran cantidad de información sobre el tema a investigar (dispositivos móviles) lo que permitió determinar la incidencia de los dispositivos móviles en la educación y sus formas de aplicación, así como también sus desventajas.

Se tomó datos estadísticos de la Unión Internacional de Telecomunicaciones (UIT) que permitió conocer el uso de dispositivos móviles a nivel mundial.

Como alternativa de búsqueda principal en la investigación se accedió a la información del Instituto Nacional de Estadísticas y Censos (INEC, 2013) de la misma manera se tuvo acceso a estadísticas de Encuesta Nacional de Empleo Desempleo y Subempleo -ENEMDU (2011

- 2013), de La Superintendencia de Telecomunicaciones, del Ministerio de Telecomunicaciones (Mintel), en base al uso de dispositivos móviles para obtener datos estadísticos que permitirán realizar una análisis profundo en cuanto a la temática de incidencia de dispositivos móviles en la educación en el Ecuador.

\section{RESULTADO DE TRABAJO}

Como resultados de trabajo a nivel descriptivo e investigativo se detalla las siguientes partes:

\section{Los dispositivos móviles y su evolución en los últimos tiempos}

Una de las líneas en la evolución de la tecnología a lo largo de los años ha consistido en fabricar nuevos dispositivos cada vez más pequeños. La tecnología ha tenido un proceso 
similar de cambio hoy que se busca la miniaturización y la portabilidad, con dispositivos más capaces y pequeños que caben en el bolsillo.

Los primeros teléfonos móviles aparecieron a finales de los años 40, después de la Segunda Guerra Mundial. Eran muy caros, pesados y de gran tamaño, por lo que en general se utilizaban dentro de los vehículos y para un público restringido. En 1982 los laboratorios Bell en Estados Unidos crearon el dispositivo que conocemos como Dynatac 8000x, pesaba 800 gramos el primer móvil a la venta también se le conoció como teléfono móvil de primera generación (1G, voz analógica) que se apoyaba en tecnología AMPS (Advanced Mobile Phone System). También se implantaron en Inglaterra y en Japón, donde se los conoció con los nombres TACS (Total Access Communications System) y MCS-L1, respectivamente (Vázquez, 2011).

En 1990 aparecieron los teléfonos de segunda generación (2G), de menor tamaño, más ligeros y con un costo menor, basados en comunicación digital tipo GSM (Global System for Mobile Communications), lo que facilitaba una transmisión de voz en alta calidad y tenían un mayor nivel de seguridad favoreciendo su integración en el mundo empresarial. A partir de ese momento, y acompañando el desarrollo de los ordenadores y de Internet (Vázquez, 2011).

Se inicia lo que se conoce como tercera generación (3G) y el desarrollo de la tecnología UMTS (Universal Mobile Telecommunications System). Los teléfonos móviles empiezan a incorporar conexión a Internet, lo que permitirá la transmisión de ficheros. Entre las funcionalidades o servicios, adquieren gran importancia las cámaras fotográficas, de video y juegos, adaptando la telefonía móvil al mundo doméstico y empresarial. Comienza entonces la verdadera revolución (Cedeño, 2011).

En la actualidad nos encontramos en la cuarta generación (4G) con transmisión de voz y datos a una mayor velocidad y se convierten en una pieza vital para el bienestar social y el mundo. La portabilidad de los dispositivos móviles, unida a una Web cada vez más rápida y la conectividad mediante teléfonos celulares, hace que los dispositivos móviles conduzcan a mejorar la productividad y el aprendizaje.

Los dispositivos móviles constituyen también un canal de distribución importante para revistas y libros digitales, lo que ha hecho que la plataforma atraiga a las mayores editoriales dedicadas a educación. 
En la actualidad nos encontramos en la de cuarta generación $(4 \mathrm{G})$ con transmisión de voz y datos a una mayor velocidad y se convierten en una pieza vital para el bienestar social y el mundo. La portabilidad de los dispositivos móviles, unida a una Web cada vez más rápida y la conectividad mediante teléfonos celulares, hace que los dispositivos móviles conduzcan a mejorar la productividad y el aprendizaje.

\section{Características generales de los dispositivos móviles}

Una gran cantidad de dispositivos electrónicos se clasifican actualmente como dispositivos móviles, desde teléfonos hasta portátiles, pasando por dispositivos como lectores de RFID1. Las características esenciales que tienen los dispositivos móviles:

- Son aparatos pequeños.

- La mayoría de estos aparatos se pueden transportar en el bolsillo del propietario o en un pequeño bolso.

- Tienen capacidad de procesamiento.

- Tienen conexión permanente o intermitente a una red.

- Tienen memoria (RAM, tarjetas MicroSD, flash, etc.).

- Normalmente se asocian al uso individual de una persona, tanto en posesión como en operación, la cual puede adaptarlos a su gusto.

- Tienen una alta capacidad de interacción mediante la pantalla o el teclado.

\section{Categorías de dispositivos móviles}

Dado el variado número de niveles de funcionalidad asociado con dispositivos móviles, en el 2005, T38 y DuPont Global Mobility Innovation Team propusieron los siguientes estándares para la definición de dispositivos móviles:

Dispositivo Móvil de Datos Limitados: son dispositivos que tienen una pantalla pequeña, principalmente basada en pantalla de tipo texto con servicios de datos generalmente limitados a SMS y acceso WAP. Un típico ejemplo de este tipo de dispositivos son los teléfonos móviles.

Dispositivo Móvil de Datos Básicos: Son dispositivos que tienen una pantalla de mediano tamaño, menú o navegación basada en íconos por medio de una rueda o cursor, y que ofrecen acceso a e-mails, lista de direcciones, SMS, y un navegador web básico. Un típico ejemplo de este tipo de dispositivos son las BlackBerry y los Teléfonos Inteligentes.

Dispositivo Móvil de Datos Mejorados: dispositivos que tienen pantallas de medianas a grandes (por encima de los 240 x 120 pixeles), navegación de tipo stylus, y que ofrecen las 
mismas características que el "Dispositivo Móvil de Datos Básicos" más aplicaciones nativas como aplicaciones de Microsoft Office Mobile y aplicaciones corporativas usuales, en versión móvil, como Sap, portales intranet, etc. Este tipo de dispositivos incluyen los Sistemas Operativos como Windows Mobile 2003 o versión 5, como en las Pocket PCs.

PocketPC es un ordenador de bolsillo, también llamado PDA (Personal Digital Assistant). Se trata de un pequeño ordenador, diseñado para ocupar el mínimo espacio y ser fácilmente transportable que ejecuta el sistema operativo Windows CE de Microsoft entre otros, el cual le proporciona capacidades similares a los PCs de escritorio (Olan, 2009).

ABI Research" (Investigaciones ABI) estima que los usuarios de dispositivos móviles descargarán, en sus teléfonos inteligentes y tabletas, 70.000 millones de Apps en el 2013 (56.000 en teléfonos y 14.000 en tabletas); esto es más de 10 Apps por cada habitante de la tierra. En un reporte especial de iLearn II llamado "Análisis de la categoría de educación en las Tiendas de Aplicaciones para Apps de Apple", anota que más del 80\% de los Apps educativos tienen como objetivo específico a los niños (L., Becker, Cummins, Estrada, \& Freeman, 2013).

\section{Funcionalidades de los dispositivos móviles y su aplicación en la educación}

Tabla 1. Funcionalidades del dispositivo móvil y su aplicación en la educación

\begin{tabular}{|c|c|c|}
\hline Funcionalidad & Application en la educación & APPS \\
\hline Para crear listas & $\begin{array}{l}\text { Para crear listas de alumnos } \\
\text { Para crear lista de tareas } \\
\text { Algunas aplicaciones se sincronizan } \\
\text { con varios dispositivos se pueden } \\
\text { compartir }\end{array}$ & $\begin{array}{ll}\text { - } & \text { Wunderlist.Busy } \\
\text { - } & \text { Astrid } \\
\text { - } & \text { gTask/google Task }\end{array}$ \\
\hline Para tomar notas a mano & $\begin{array}{l}\text { Sirve para añadir contendido en } \\
\text { formas de notas } \\
\text { Algunas aplicaciones se sincronizan } \\
\text { con varios dispositivos se pueden } \\
\text { compartir y enviar por mail } \\
\text { Mejora la productividad }\end{array}$ & $\begin{array}{l}\text { - Evernote } \\
\text { - Colornote } \\
\text { - Google keep } \\
\text { - Plaintext } \\
\text { - Notz }\end{array}$ \\
\hline Para gestión de la clase & $\begin{array}{l}\text { Sirve a modo de libreta del profesor } \\
\text { Pueden incluirse fichas, calendarios, } \\
\text { calificaciones, notas } \\
\text { Algunas aplicaciones permiten la } \\
\text { exportación de Excel }\end{array}$ & $\begin{array}{l}\text { - Homework } \\
\text { - Idoceo } \\
\text { - Classtime Schedule } \\
\text { - Teacher kit } \\
\text { - Cuaderno del profesor }\end{array}$ \\
\hline Para crear contenidos & $\begin{array}{l}\text { Para hacer textos } \\
\text { Hacer mapas } \\
\text { Hacer posters } \\
\text { Para añadir video, fotos }\end{array}$ & $\begin{array}{ll}\text { - } & \text { Movenote } \\
\text { - } & \text { Socrative } \\
\text { - } & \text { Thinglink } \\
\text { - } & \text { Blog }\end{array}$ \\
\hline
\end{tabular}




\section{Aprendizaje móvil o móvil learning}

Según (Fumero, Reverón, 2010), el Mobile Learning es una "ESCUELA EN EL BOLSILLO”.

Según (O’Malley et al. (2003), el Mobile Learning es “cualquier tipo de aprendizaje que se produce cuando el alumno no se encuentra en una ubicación fija y predeterminada; o de aprendizaje que se produce cuando el alumno se aprovecha de las oportunidades de aprendizaje que ofrecen las tecnologías móviles".

Según Mike Sharples, “el aprendizaje móvil puede ser definido como los procesos para alcanzar el conocimiento a través de la exploración y de la conversación en múltiples contextos entre las personas y con tecnologías interactivas".

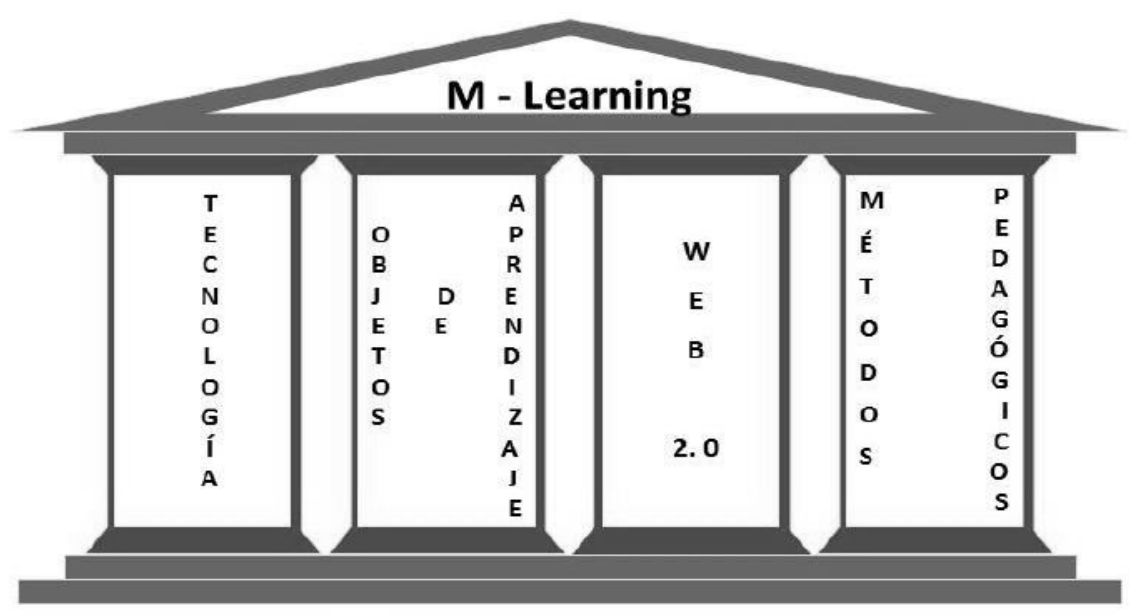

Figura 1. Bases del M-learning

Fuente:http://www.repositoriodigital.ipn.mx/bitstream/handle/123456789/53 95/521.pdf?sequence $=1$

Quinn (2000), afirma que el Mobile Learning es eLearning a través de dispositivos Computacionales móviles: Dispositivos Asistentes Personales (Personal Digital Assistant, PDA), Máquinas Windows (Entre ellos los computadores de mano, los computadores portátiles o Laptop's y los Table PC) y teléfonos celulares.

El mobile learning es la intersección de la computación móvil y el eLearning, la cual se caracteriza por la capacidad de acceder a recursos de aprendizaje desde cualquier lugar, en cualquier momento, con altas capacidades de búsqueda, alta interacción, alto soporte para 
un aprendizaje efectivo y una constante valoración basada en el desempeño (Valenzuela Sosa, 2012).

Lo que caracteriza al m-learning es que se refiere a los ambientes de aprendizaje basados en la tecnología móvil, orientados a optimizar un aprendizaje relacionado con el u-learning (Ubiquitous Learning, o aprendizaje ubicuo), concepto que hace referencia al aprendizaje apoyado en la tecnología y que se puede realizar en cualquier momento y desde cualquier lugar. Este aprendizaje ofrece métodos modernos de apoyo al proceso de aprendizaje, mediante el uso de instrumentos móviles como: las computadoras portátiles y las tables, los lectores Mp3, los teléfonos inteligentes (smartphones) y teléfonos móviles (Trabaldo, Santiago, \& Kamijo, 2015).

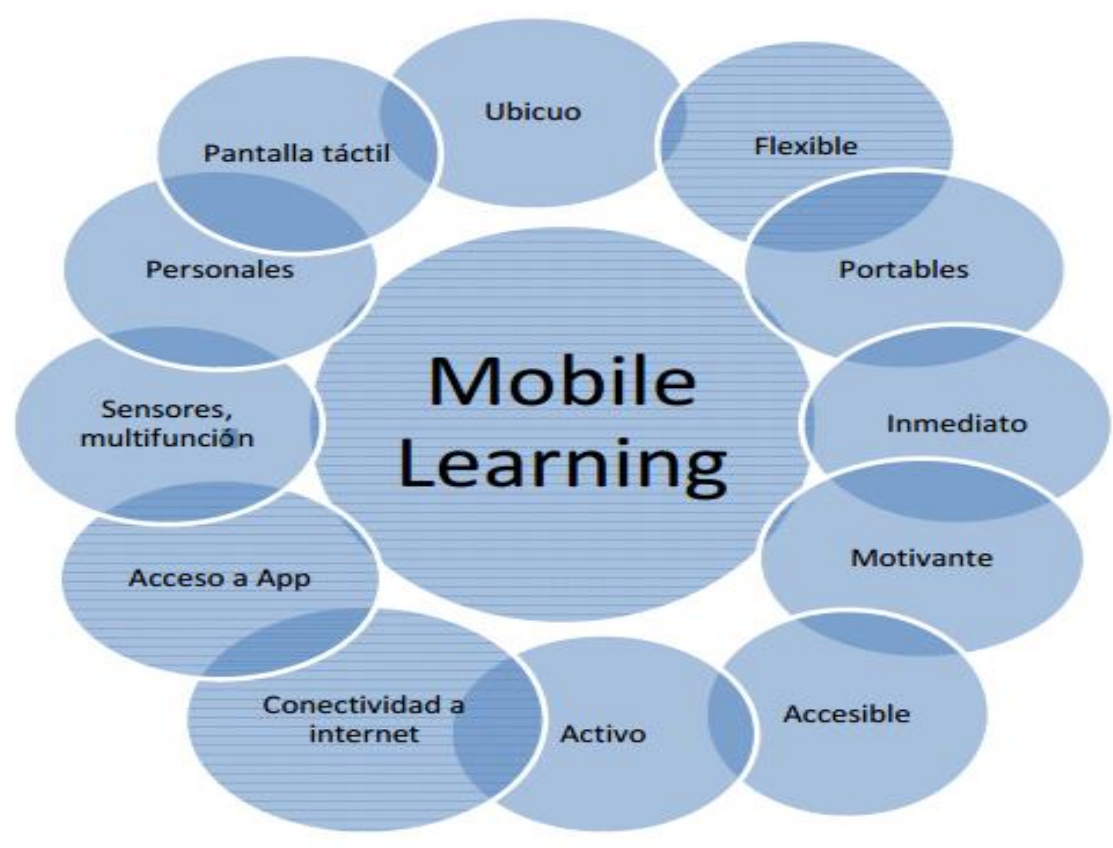

Figura 2: Características del M-learning

Fuente: http://serviciosgate.upm.es/docs/asesoramiento/guia_implementacion_movil.pdf

\section{Ventajas pedagógicas}

- Permite a los docentes tengan mayor interacción con los alumnos ya que pueden enviar recordatorios de tareas, así como mensajes de estímulo estar en contacto permanente por medio de redes sociales.

- Ayuda a combatir la resistencia al uso de las TIC y pueden ayudar a tender un puente sobre la brecha entre la alfabetización a través del teléfono móvil. 
- Beneficia a los estudiantes durante periodos extensos de clases ya que ayuda a que los alumnos estén enfocados y calmados.

- Comunicación en tiempo real con estudiantes, docentes, padres de familia y directivos.

- Evaluación y medición de respuestas, evaluaciones académicas a distancia, investigaciones y resultados en forma inmediata.

- La telefonía móvil está al alcance de casi todos, puesto que es más barata que las computadoras convencionales

- Portabilidad (dada por el tamaño y peso del dispositivo)

- Permiten capturar pensamientos e ideas en el momento que se presentan.

\section{Desventajas pedagógicas}

- Con excepción de los tablet PC y los portátiles, presentan problemas asociados a la usabilidad, debido a las pequeñas pantallas.

- Existen pocas aplicaciones educativas para los dispositivos móviles. La industria está plagada de soluciones propietarias y los costos de acceso a la red son altos, sin embargo, la industria de software ha visto que hay un interesante nicho de mercado que debe cubrirse.

- Resistencia al cambio por parte de los estudiantes o docentes

- Dificultad de adaptación a los dispositivos móviles

- La navegación suele ser limitada

- Los costos de acceso a la red son altos

- El trabajo colaborativo es menor

- La Necesidad de conexión inalámbrica que se puede ver afectada por la ubicación física del usuario (Ponce, 2010).

\section{Implantar M-learning en la educación}

Este proceso de enseñanza tiene una serie de repercusiones que no nos ofrece la enseñanza presencial, tal y como indica (Barberá. E., 2003)

" se extienden al menos en cuatro direcciones: la primera, temporalmente, en cuanto dilata el tiempo educativo puesto que no se debe ceñir a las horas de clase; la segunda, geográficamente, en cuanto se ensancha el radio de acción educativa, dado que puede 
llegar a alumnos dispersos por territorios cercanos o lejanos; la tercera, cognitivamente, atendiendo a habilidades que docentes $\mathrm{y}$ estudiantes pueden desarrollar de manera diferente con el uso de procesos facilitados por la tecnología y la cuarta, en relación con los recursos, puesto que tanto alumnos como profesores pueden disfrutar de un conjunto casi ilimitado de fuentes documentales de todo tipo" (Barberá. E, 2003).

Es decir, este autor nos habla del tiempo, puesto que el proceso de enseñanza - aprendizaje está abierto las 24 horas, todos los días del año; porque podemos estar estudiando mientras nos trasladamos a distintos lugares; el cambio en la mentalidad de docente puesto que conlleva un cambio en la filosofía del aprendizaje, ya no se centra tanto en el profesor hablando y el alumno escuchando, sino en el alumno organizando su proceso de formación y el profesor orientando dicho proceso, como un guía.

Para ello, seguiremos al autor Guerrero, E. (2006), que establece como requisitos para la implementación del móvil learning:

- Eliminar el control al usuario

- Automatizar el servicio tanto como sea posible.

- Mantener los procesos de configuración un número mínimo de pasos.

- Mantener direcciones necesarias para ayuda e información.

- Proveer toda la información necesaria para el usuario.

- Proveer de toda la información de configuración en el lenguaje y vocabulario del usuario.

- Permitir el error humano

- Permitir acceso a información de configuración durante los procedimientos de configuración.

- Utilizar estándares y guías existentes.

- Diseñar servicios y soluciones para diferentes capacidades de los usuarios.

Es decir, enseñar al usuario un mínimo de uso de todos los elementos necesarios para este proceso de enseñanza - aprendizaje, para que no pierda la motivación y pueda valerse por sí mismo (Guerrero, 2011). 


\section{Descripción estadística del uso de dispositivos móviles en América Latina}

Como resultado de las estadísticas a nivel de américa latina a finales del 2014 por la firma Según un estudio de GuiaLocal.com se obtuvieron los siguientes datos:

\begin{tabular}{|c|c|c|c|c|}
\hline Puesto & Pais & Celulares $\%$ & Tablets $\%$ & Total Móvil \% \\
\hline $\mathbf{1}$ & Nicaraqua & 138 & 54 & 123 \\
\hline $\mathbf{2}$ & Colombia & 133 & 48 & 115 \\
\hline $\mathbf{3}$ & Guatemala & 108 & 17 & 100 \\
\hline $\mathbf{4}$ & Arqentina & 100 & 48 & 92 \\
\hline $\mathbf{5}$ & Ecuador & 76 & 75 & 76 \\
\hline $\mathbf{6}$ & Chile & 77 & 64 & 76 \\
\hline $\mathbf{7}$ & Rep. & & & 73 \\
\hline $\mathbf{8}$ & Dominicana & 84 & 13.43 & 67 \\
\hline $\mathbf{9}$ & Menduras & 76 & 19 & 55 \\
\hline $\mathbf{1 0}$ & Venezuela & 39 & 20 & 33 \\
\hline 11 & Brasil & 7.50 & 14.49 & 9 \\
\hline
\end{tabular}

Figura 3. El ranking de países que mayor crecimiento en el uso de dispositivos móviles

Fuente: http://gestion.pe/tecnologia/uso-dispositivos-moviles-crecio-61-america-latina2121429

En la figura 3 se nota que en año 2014 en América Latina el uso de dispositivos móviles creció $61 \%$, mientras que el uso de computadores para conectarse a Internet registra una caída de $11.3 \%$.

El ranking de países que mayor crecimiento mostraron en el uso de dispositivos móviles es liderado por Nicaragua, que registra un crecimiento anual del 123\%. Países como Guatemala, Ecuador y Honduras han sorprendido con su crecimiento

\section{Estadísticas a nivel nacional ecuador}

Ecuador cuenta con más de 13 millones de líneas celulares asignadas entre tres operadoras, con las cuales cerca del $10 \%$ de usuarios acceden constantemente a Internet según la Superintendencia de Telecomunicaciones hasta principios de 2010. 


\begin{tabular}{|l|c|c|c|c|}
\hline \multicolumn{5}{|c}{ Tenencia de celular : Nacional } \\
\hline & 2008 & 2009 & 2010 & 2011 \\
\hline Pichincha & $51,6 \%$ & $55,7 \%$ & $57,4 \%$ & $55,0 \%$ \\
\hline Guayas & $39,6 \%$ & $46,9 \%$ & $46,1 \%$ & $50,6 \%$ \\
\hline El Oro & $39,7 \%$ & $40,4 \%$ & $44,6 \%$ & $50,3 \%$ \\
\hline Azuay & $38,8 \%$ & $42,7 \%$ & $47,4 \%$ & $47,8 \%$ \\
\hline Santo Domingo & - & - & - & $47,8 \%$ \\
\hline Total Nacional & $21,4 \%$ & $38,8 \%$ & $23,9 \%$ & $46,6 \%$ \\
\hline Loja & $29,7 \%$ & $36,1 \%$ & $40,7 \%$ & $46,4 \%$ \\
\hline Los Ríos & $32,9 \%$ & $34,5 \%$ & $39,2 \%$ & $46,2 \%$ \\
\hline Imbabura & $34,9 \%$ & $37,0 \%$ & $40,8 \%$ & $45,4 \%$ \\
\hline Tungurahua & $34,5 \%$ & $38,4 \%$ & $41,3 \%$ & $45,3 \%$ \\
\hline Manabí & $30,2 \%$ & $32,7 \%$ & $35,1 \%$ & $44,1 \%$ \\
\hline Santa Elena & - & - & $43,4 \%$ & $42,4 \%$ \\
\hline Carchi & $32,4 \%$ & $36,0 \%$ & $34,9 \%$ & $40,2 \%$ \\
\hline Esmeraldas & $29,1 \%$ & $31,0 \%$ & $32,2 \%$ & $40,0 \%$ \\
\hline Cañar & $29,2 \%$ & $31,2 \%$ & $33,9 \%$ & $38,5 \%$ \\
\hline Cotopaxi & $27,0 \%$ & $29,2 \%$ & $30,0 \%$ & $33,9 \%$ \\
\hline Amazonía & - & $24,9 \%$ & $33,6 \%$ & $31,7 \%$ \\
\hline Chimborazo & $24,9 \%$ & $27,4 \%$ & $28,6 \%$ & $30,5 \%$ \\
\hline Bolívar & $22,4 \%$ & $24,9 \%$ & $25,1 \%$ & $29,7 \%$ \\
\hline
\end{tabular}

Figura 4: Tenencia de un celular Ecuador

Fuente: http://www.inec.gob.ec/sitio_tics/presentacion.pdf

Como se puede observar en los resultados se indica que en la provincia de Pichincha el 55\% de personas tienen un celular activado, seguido de la provincia del Guayas con el 50,6\%. mientras la provincia de Bolívar registra el menor porcentaje con el 29,7\%

Ecuador pasó del 8 al 16\% de personas que tienen acceso a un Smartphone o teléfono inteligente. Esta es el último dato proporcionado por la Encuesta de Tecnologías de la Información y la Comunicación (TIC) del Instituto Nacional de Estadística y Censos (INEC), en el Ecuador según estadísticas aproximadamente el $80 \%$ de hogares poseen un teléfono celular.

\section{CONCLUSIONES}

- De acuerdo con la bibliografía encontrada se puede deducir que existe investigación sobre los dispositivos móviles a nivel nacional, lo cual ha permitido analizar que el Ecuador ocupa el 5to lugar en las estadísticas de usuarios de dispositivos móviles en 
América latina lo que denota que hay un crecimiento tecnológico según estadísticas aproximadamente el $80 \%$ de hogares poseen un teléfono celular.

- Se puede evidenciar que en el año 2014 en América Latina el uso de dispositivos móviles creció $61 \%$, mientras que el uso de computadores para conectarse a Internet registra una caída de $11.3 \%$.

- El ranking de países que mayor crecimiento mostraron en el uso de dispositivos móviles es liderado por Nicaragua, que registra un crecimiento anual del $123 \%$. Países como Guatemala, Ecuador y Honduras han sorprendido con su crecimiento

- Otra conclusión importante que se determinó es que el 29,1\% de la población utiliza los dispositivos móviles para el aprendizaje y la educación según los últimos datos del Instituto Nacional de Estadística y Censos (INEC 2013).

\section{REFERENCIAS BIBLIOGRÁFICAS}

Cantillo Valero, C., Roura Redondo, M., \& Sánchez Paladín, M. (2012). Tendencias actuales en uso de dispositivos móviles en la educación. la educación, 21.

Cedeño, E. (2011). Evolución y Revolución de la Telefonía. Recuperado el 11 de febrero de 2016, de http://www.digibis.com/digibibdemo/es/catalogo_imagenes/grupo.cmd?path=1000080

Gestión, P. (23 de enero de 2015). Recuperado el 14 de febrero de 2016, de Uso de dispositivos móviles creció $61 \%$ en América Latina: http://gestion.pe/tecnologia/uso-dispositivos-moviles-crecio-61-america-latina2121429

Guaña-Moya, J. Q.-A.-T. (2018). Estudio preliminar del uso de las redes sociales en los jóvenes ecuatorianos. Revista PUCE, 106.

Guerrero, A. J. (17 de diciembre de 2011). Móvil E-learning. Recuperado el 12 de febrero de 2016, de http://recursostic.educacion.es/observatorio/web/fr/cajon-de-sastre/38cajon-de-sastre/1026-movil-learning

INEC. (16 de mayo de 2014). INEC. Recuperado el 8 de febrero de 2016, de http://www.ecuadorencifras.gob.ec/12-millones-de-ecuatorianos-tienen-untelefono-inteligente-smartphone/

Infobae. (27 de diciembre de 2011). El celular, el dispositivo móvil más usado.

Recuperado el 14 de febrero de 2016, de http://www.infobae.com/2011/12/27/1040947-el-celular-el-dispositivo-movil-masusado 
L., H., Becker, A., Cummins, S., Estrada, M., \& Freeman, A. (1 de diciembre de 2013). EDUTEKA. Recuperado el 16 de febrero de 2016, de APRENDIZAJE MEDIANTE DISPOSITIVOS MÓVILES: http://www.eduteka.org/articulos/dispositivosmoviles

LOEI. (2008). Ministerio de Educación. Recuperado el 7 de febrero de 2016, de http://educacion.gob.ec/ley-organica-de-educacion-intercultural-loei/

Luna-Echeverría, N. E.-L.-H.-M. (2018). Uso de las Tecnológicas de la Información y Comunicación (TIC) en los Institutos de Educación Superior. Polo del Conocimiento, 300-315.

MINEDUC. (14 de mayo de 2014). Ministerio de Educación. Recuperado el 14 de febrero de 2016, de http://educacion.gob.ec/mineduc-expide-regulaciones-para-el-uso-detelefonos-celulares-en-instituciones-educativas/

Moreno, A. J. (8 de febrero de 2013). Observatorio Tecnológico. Recuperado el 14 de febrero de 2016, de ¿Cómo implementar el e-learning en los procesos de enseñanza-aprendizaje?:

http://recursostic.educacion.es/observatorio/web/ca/internet/recursos-online/1089icomo-implementar-el-e-learning-en-los-procesos-de-ensenanza-aprendizaje

Olan, J. R. (25 de junio de 2009). Slideshare. Recuperado el 15 de febrero de 2016, de http://es.slideshare.net/DACB_Lcc/dispositivos-moviles-1639336

Pérez-Fabara, M. A.-A.-A. (2017). Las tecnologías en el mejoramiento de los procesos educativos en la Educación Superior en América Latina. Revista Publicando, 704718.

Ponce, J. P. (3 de mayo de 2010). Formación Gerencial. Recuperado el 14 de febrero de 2016, de Tecnología Móvil en la Educación. Aplicaciones, usos y tendencias: http://blog.formaciongerencial.com/2010/05/03/tecnologia-movil-en-la-educacionaplicaciones-usos-y-tendencias/

Trabaldo, S., Santiago, R., \& Kamijo, M. (2015). Mobile E-learning nuevas realidades en el aula. España: Océano.

UNESCO. (2013). Directrices para las políticas de aprendizaje Móvil. Recuperado el 13 de febrero de 2016, de http://www.unesco.org/new/fileadmin/MULTIMEDIA/HQ/ED/ICT/images/114_13 _ED_UNESCO_Policy_Guidelines_for_Mobile_Learning_S.pdf

Valenzuela Sosa, M. (13 de marzo de 2012). Dispositivos Móviles en Educación. Recuperado el 16 de febrero de 2016, de Dispositivos Móviles en Educación: http://es.slideshare.net/Mvalenzuelasosa/dispositivos-mviles-en-educacin

Vázquez, N. A. (2011). Información en el Móvil. En N. A. Vázquez, Información en el Móvil (págs. 13-16). Barcelona: UOC. 


\section{PARA CITAR EL ARTÍCULO INDEXADO.}

Terán Acosta, G., Oña Gamboa, V., Cobos Velasco, J., \& Miniguano Miniguano, D. (2019). Incidencia de dispositivos móviles en la educación en el Ecuador. Ciencia Digital, 3(3.4.), 60-74. https://doi.org/10.33262/cienciadigital.v3i3.4.835

\section{\Ciencia}

El artículo que se publica es de exclusiva responsabilidad de los autores y no necesariamente reflejan el pensamiento de la Revista Ciencia Digital.

El artículo queda en propiedad de la revista y, por tanto, su publicación parcial y/o total en otro medio tiene que ser autorizado por el director de la Revista Ciencia Digital.
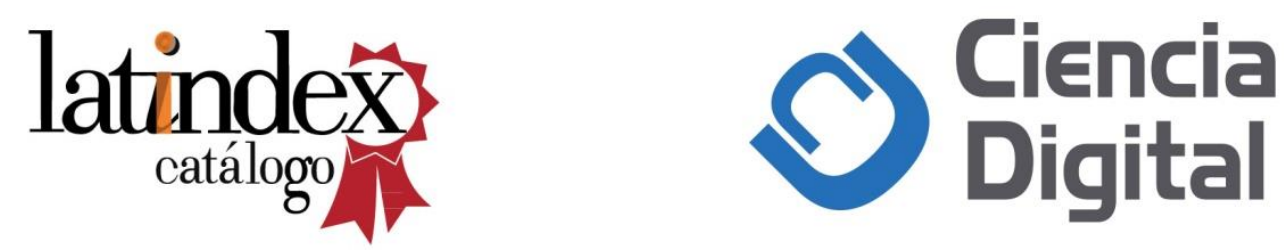